\title{
BOUNDARY ALGEBRAIC EQUATIONS FOR LATTICE PROBLEMS
}

\author{
P.G. MARTINSSON AND G.J. RODIN
}

\begin{abstract}
Boundary algebraic equations corresponding to Dirichlet boundary-value problems on lattices are introduced. These equations are based on the lattice Green's function, from which discrete single- and double-layer potentials are derived. Structurally, the boundary algebraic equations are similar to the boundary integral equations of classical potential theory. Numerical experiments indicate that boundary algebraic equations possess excellent spectral properties.
\end{abstract}

\section{INTRODUCTION}

In this paper, we present a new method for solving conduction problems defined on periodic lattices of finite extent. Conceptually, the new method is similar to the boundary integral equation methods of classical potential theory (Mikhlin, 1957). In particular, in the new method, the discrete boundary-value problems defined on lattices are re-formulated in terms of equivalent boundary algebraic (as opposed to integral) equations that involve lattice Green's functions as the kernels. As a result one replaces sparse algebraic problems defined on the entire domain with dense algebraic problems defined on the domain boundary only. This replacement is particularly useful for very large problems because (i) the dense algebraic problems involve less unknowns, (ii) they can be solved using $\mathcal{O}(N)$ summation methods, and (iii) they involve much smaller condition numbers than the sparse algebraic problems defined on the entire domain.

Due to the space constraints, we restrict our attention to Dirichlet problems defined for twodimensional structures made of square lattices. For these problems, we develop boundary equations involving the discrete single- and double-layer kernels and present several example problems which support the notion that boundary algebraic equations posses superior spectral properties. For further details, we refer to Martinsson (2002), who also provides a detailed treatment of lattice Green's functions and the corresponding $\mathcal{O}(N)$ summation methods.

The paper is organized as follows. In Section 2, we introduce the notation and define the discrete Dirichlet boundary-value problem. In Section 3, we introduce the discrete single- and double-layer kernels and construct the boundary algebraic equations for the homogeneous Dirichlet boundaryvalue problem. In Section 4, we generalize the boundary algebraic equations to inhomogeneous problems and irregular lattices. In Section 5, we present numerical examples that demonstrate the superior spectral properties of the boundary algebraic equations.

\section{Problem Statement}

Consider the lattice obtained by connecting each node in $\mathbb{Z}^{2}$ to its four nearest neighbors by links of conductivity one. Poisson's equation for heat conduction on this lattice reads

$$
[\mathfrak{A} \mathfrak{u}](m)=\mathfrak{f}(m), \quad \forall m \in \mathbb{Z}^{2},
$$




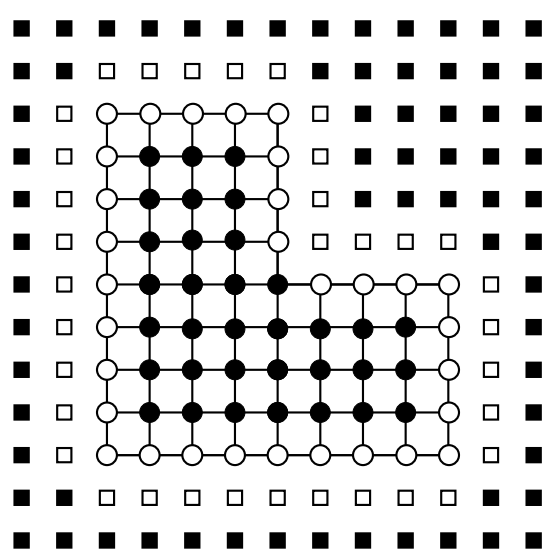

(a)

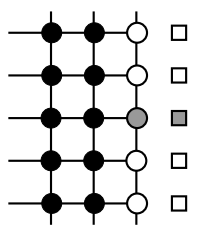

(b)

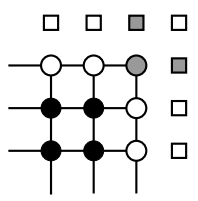

(c)

Figure 1. (a) An example of a lattice domain, $\Omega=\Gamma \cup \Omega_{-}$. The black circles form the interior $\Omega_{-}$and the white circles form the boundary $\Gamma$. (b) Illustration of the set $\mathbb{D}_{n}$ (the grey square) for a boundary node $n$ (grey circle) along a straight edge.

(c) Illustration of $\mathbb{D}_{n}$ for a corner node.

where $\mathfrak{u}(m)$ is the unknown temperature and $\mathfrak{f}(m)$ is a prescribed heat source. The discrete Laplace operator is defined by

$$
[\mathfrak{A} \mathfrak{u}](m)=4 \mathfrak{u}(m)-\mathfrak{u}\left(m+e_{1}\right)-\mathfrak{u}\left(m-e_{1}\right)-\mathfrak{u}\left(m+e_{2}\right)-\mathfrak{u}\left(m-e_{2}\right),
$$

where $e_{1}=[1,0]$ and $e_{2}=[0,1]$.

Next we consider heat conduction on the finite lattice obtained by connecting the nodes in a finite subset $\Omega \subset \mathbb{Z}^{2}$, see Fig. 1a. The boundary of $\Omega$ is denoted by $\Gamma$ and is defined as the set of points with less than four neighbors in $\Omega$. With the introduction of the set of interior nodes $\Omega_{-}:=\Omega \backslash \Gamma$, the discrete homogeneous Dirichlet problem reads

$$
\left\{\begin{aligned}
{[\mathfrak{A} \mathfrak{u}](m) } & =0, & & m \in \Omega_{-}, \\
\mathfrak{u}(m) & =\mathfrak{g}(m), & & m \in \Gamma,
\end{aligned}\right.
$$

where $\mathfrak{g}(m)$ are prescribed nodal temperatures.

\section{Single And Double Layer Kernels}

Using Fourier analysis, it is possible to derive a fundamental solution of (1) in the form

$$
\mathfrak{G}(m, n)=\frac{1}{(2 \pi)^{2}} \int_{(-\pi, \pi)^{2}} \frac{e^{-\mathbf{i}(m-n) \cdot \xi}-1}{4 \sin ^{2} \frac{\xi_{1}}{2}+4 \sin ^{2} \frac{\xi_{2}}{2}} d \xi .
$$

This function satisfies the following equation

$$
[\mathfrak{A} \mathfrak{G}](m, n)=\left\{\begin{array}{ll}
1 & \text { if } m=n \\
0 & \text { if } m \neq n
\end{array} \quad m, n \in \mathbb{Z}^{2} .\right.
$$

When $|m-n|$ is large, the function $\mathfrak{G}$ closely approximates the fundamental solution of the Laplace operator, in fact $\mathfrak{G}(m, n)=-(2 \pi)^{-1} \log |m-n|+O\left(|m-n|^{-2}\right)$ as $|m-n| \rightarrow \infty$, see Duffin (1953). 
However, the behavior is quite different when $|m-n|$ is small, for instance, $\mathfrak{G}(m, m)$ is finite. As far as boundary algebraic equations are concerned, $\mathfrak{G}(m, n)$ is the single-layer kernel.

In order to define a discrete analogue of the double layer potential, we envision $\Omega$ to be embedded in the infinite lattice $\mathbb{Z}^{2}$. The double layer kernel $\mathfrak{G}_{\nu}(m, n)$ is then defined as the flux through the boundary $\Gamma$ at the boundary node $n$ that is induced by a point charge at node $m$. Letting $\mathbb{D}_{n}$ denote the nodes in $\mathbb{Z}^{2} \backslash \Omega$ that connect to the node $n$ (see Fig. 1b,c), we can write

$$
\mathfrak{G}_{\nu}(m, n)=\sum_{k \in \mathbb{D}_{n}} \mathfrak{G}(m, k)-\mathfrak{G}(m, n) .
$$

The double layer kernel could alternatively be defined by first introducing an exterior difference operator $\partial_{\nu}$ by setting $\partial_{\nu} \mathfrak{u}(n)=\sum_{k \in \mathbb{D}_{n}}(\mathfrak{u}(k)-\mathfrak{u}(n))$ and then defining $\mathfrak{G}_{\nu}(m, n)=\partial_{\nu_{n}} \mathfrak{G}(m, n)$.

\section{Boundary Algebraic Equations}

4.1. Indirect Boundary Algebraic Equations. In order to construct an indirect boundary algebraic equation corresponding to (2) we make the ansatz

$$
\mathfrak{u}(m)=\sum_{n \in \Gamma} \mathfrak{G}(m, n) \phi(n) .
$$

Then automatically, $[\mathfrak{A} \mathfrak{u}](m)=0$ for $m \in \Omega_{-}$. The boundary condition is satisfied if the sources $\phi(n)$ are chosen so that

$$
\sum_{n \in \Gamma} \mathfrak{G}(m, n) \phi(n)=\mathfrak{g}(m) \quad \forall m \in \Gamma
$$

This formulation is characterized by a positive definite matrix (Martinsson, 2002) and therefore has a unique solution for any $\mathfrak{g}$. In contrast, the integral equation corresponding to (4) is of the first kind, and uniqueness of its solution is not generally guaranteed.

Alternatively, we can use the double layer kernel in the ansatz,

$$
\mathfrak{u}(m)=\sum_{n \in \Gamma} \mathfrak{G}_{\nu}(m, n) \phi(n),
$$

which leads to the algebraic equation

$$
\sum_{n \in \Gamma} \mathfrak{G}_{\nu}(m, n) \phi(n)=\mathfrak{g}(m) \quad \forall m \in \Gamma .
$$

Here $\phi(n)$ represents a layer of dipoles formed by placing couples of heat sources of opposite signs on the boundary of $\Omega$ and the boundary of $\Omega^{\mathrm{c}}$ (the white nodes in Fig. 1a). The spectral properties of (5) are similar to those of the discretization of the corresponding second kind integral equation.

4.2. Direct Boundary Algebraic Equations. To formulate a direct boundary algebraic equation corresponding to (2), let us consider two thermal states of $\Omega$. The first state involves the nodal temperatures $\mathfrak{u}$ and boundary fluxes $\mathfrak{u}_{\nu}$ corresponding to the solution of (2); of course at this stage only the nodal temperatures on $\Gamma$ are known. The second state involves the nodal temperatures and fluxes generated by imbedding $\Omega$ within an infinite lattice and placing a unit heat source at a 
point $m$ on $\Gamma$. These nodal temperatures and fluxes are $\mathfrak{G}(n, m)$ and $\mathfrak{G}_{\nu}(m, n)$, respectively. Then by applying the reciprocity theorem to these two states we obtain:

$$
\mathfrak{u}(m)+\sum_{n \in \Gamma} \mathfrak{G}_{\nu}(m, n) \mathfrak{u}(n)=\sum_{n \in \Gamma} \mathfrak{G}(n, m) \mathfrak{u}_{\nu}(n) .
$$

By imposing the boundary condition and replacing $\mathfrak{G}(n, m)$ with $\mathfrak{G}(m, n)$, we obtain a direct boundary algebraic equation

$$
\sum_{n \in \Gamma} \mathfrak{G}(m, n) \mathfrak{u}_{\nu}(n)=\mathfrak{g}(m)+\sum_{n \in \Gamma} \mathfrak{G}_{\nu}(m, n) \mathfrak{g}(n) \quad \forall m \in \Gamma
$$

As expected, this equation is similar to the indirect equation based on the single-layer kernel.

Remark: We defined the boundary is such a way that it does not include a vertex node at a $270^{\circ}$ angle, such as the re-entrant corner node in Fig. 1a. If it is desired to prescribe boundary conditions at such a node, this can easily be done by including an additional unknown for each such node in the system of boundary equation. We have found that such en enrichment of the system does not significantly change the conditioning.

\section{INCLUSIONS}

The methods presented in Section 4.1 can be extended to analysis of lattices with inclusions. To this end, let us consider the perturbed Dirichlet problem

$$
\left\{\begin{aligned}
{\left[\left(\mathfrak{A}-\mathfrak{A}_{\mathrm{r}}\right) \mathfrak{u}\right](m) } & =0, & & m \in \Omega_{-}, \\
\mathfrak{u}(m) & =\mathfrak{g}(m), & & m \in \Gamma,
\end{aligned}\right.
$$

where $\mathfrak{A}_{\mathrm{r}}$ represents a (typically local) perturbation due to inclusions. The first step is to reformulate (7) as an unperturbed problem

$$
\left\{\begin{aligned}
{[\mathfrak{A} \mathfrak{u}](m) } & =\mathfrak{f}_{\mathrm{r}}, & & m \in \Omega_{-}, \\
\mathfrak{u}(m) & =\mathfrak{g}(m), & & m \in \Gamma,
\end{aligned}\right.
$$

where $\mathfrak{f}_{\mathrm{r}}=\mathfrak{A}_{\mathrm{r}} \mathfrak{u}$ are fictitious heat sources applied to the unperturbed lattice. For concreteness, let us suppose that $\mathfrak{A}_{\mathrm{r}}$ represents $J$ removed bars. The nodes of the $j$-th bar are denoted $k_{-}^{(j)}$ and $k_{+}^{(j)}$. Now, there exists a $\psi(j)$ (to be determined), such that the effect of removing the bar $j$ is identical to adding charges $-\psi(j)$ and $+\psi(j)$ at the nodes $k_{-}^{(j)}$ and $k_{+}^{(j)}$ to the unperturbed lattice. Thus

$$
\mathfrak{f}_{\mathrm{r}}(m)=\sum_{j=1}^{J} \psi(j)\left[\delta\left(m, k_{+}^{(j)}\right)-\delta\left(m, k_{-}^{(j)}\right)\right],
$$

where $\delta$ is the Kronecker symbol. With a single layer potential on the boundary, the ansatz is now

$$
\mathfrak{u}(m)=\sum_{n \in \Gamma} \mathfrak{G}(m, n) \phi(n)+\sum_{j=1}^{J}\left[\mathfrak{G}\left(m, k_{+}^{(j)}\right)-\mathfrak{G}\left(m, k_{-}^{(j)}\right)\right] \psi(j) .
$$

To determine $\phi$ and $\psi$ we first invoke the boundary condition,

$$
\mathfrak{g}(m)=\sum_{n \in \Gamma} \mathfrak{G}(m, n) \phi(n)+\sum_{j=1}^{J}\left[\mathfrak{G}\left(m, k_{+}^{(j)}\right)-\mathfrak{G}\left(m, k_{-}^{(j)}\right)\right] \psi(j), \quad m \in \Gamma .
$$



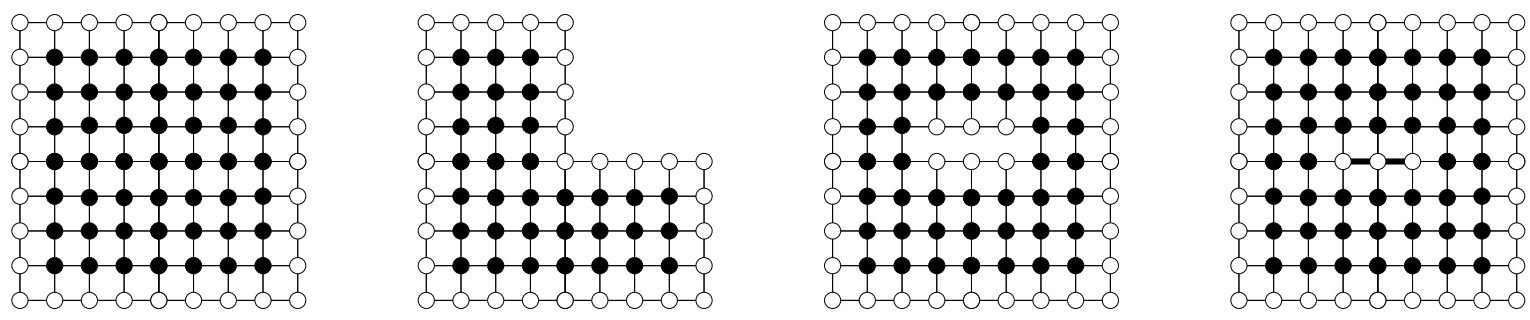

Figure 2. The geometries we used to estimate the condition numbers. Reading from left to right, first row first, we label them: "the square", "the L-shape", "the slit" and "the shortcut". The long side of the square has $2 N+1$ nodes.

Then we get $J$ additional conditions by requiring that the flux through the removed bar $i$ equals $\psi(i)$, which is to say that $\psi(i)=\mathfrak{u}\left(k_{+}^{(i)}\right)-\mathfrak{u}\left(k_{-}^{(i)}\right)$, or

$$
\begin{aligned}
\psi(i)=\sum_{n \in \Gamma}\left[\mathfrak{G}\left(k_{+}^{(i)}, n\right)-\right. & \left.\mathfrak{G}\left(k_{-}^{(i)}, n\right)\right] \phi(n)+ \\
& \sum_{j=1}^{J}\left[\mathfrak{G}\left(k_{+}^{(i)}, k_{+}^{(j)}\right)-\mathfrak{G}\left(k_{+}^{(i)}, k_{-}^{(j)}\right)-\mathfrak{G}\left(k_{-}^{(i)}, k_{+}^{(j)}\right)+\mathfrak{G}\left(k_{-}^{(i)}, k_{-}^{(j)}\right)\right] \psi(j) .
\end{aligned}
$$

Combined, equations (10) and (11) uniquely determine the heat sources $\phi$ and $\psi$.

A double layer equation can be obtained by simply replacing $\mathfrak{G}(m, n)$ in $(9)$ by the double layer kernel $\mathfrak{G}_{\nu}(m, n)$. An alternative technique for deriving equations that govern lattices with inclusions is to use the direct methods of Section 4.2. One will find that the sums in the reciprocity theorems will include the nodes $k_{ \pm}^{(j)}$ and that the resulting equations are very similar to the ones derived above.

\section{Conditioning of the Boundary EQUations}

In this section we will present several examples that indicate that the conditioning of the boundary algebraic equations is superior to that of the difference equation (1). Furthermore, as far as conditioning is concerned, we show that the boundary algebraic equations are at least as good as discretized integral equations. To this end we consider Dirichlet problems on the four domains shown in Figure 2, namely:

- A square with $(2 N+1) \times(2 N+1)$ nodes.

- A square with one quadrant removed, i.e. an L-shaped domain.

- A square with a horizontal slit extending along the middle third of the middle row.

- A square with a horizontal shortcut extending along the middle third of the middle row.

We let $K, K_{\mathrm{S}}$ and $K_{\mathrm{D}}$ denote the matrices associated with the original difference equation, the single formulation, and the double layer formulation, respectively. For each geometry, we computed the condition numbers for these matrices for $N$ in the range between 2 and 50. Results of the computation are summarized in Table 1.

The numbers in the table lend support to our belief that the conditioning of boundary algebraic equations is superior to that of the original difference equations. Further, the condition numbers for the square are consistent with the condition numbers for discretized integral equations, see Atkinson 


\begin{tabular}{c|cccc} 
& Square & L-shape & Slit & Shortcut \\
\hline $\operatorname{cond}(K)$ & $1.6 N^{2}$ & $0.83 N^{2}$ & $1.6 N^{2}$ & $0.28 N^{3}$ \\
$\operatorname{cond}\left(K_{\mathrm{S}}\right)$ & $40 N$ & $40 N$ & $4 N^{2}$ & $4.2 N^{2}$ \\
$\operatorname{cond}\left(K_{\mathrm{D}}\right)$ & 7.0 & 7.3 & $0.63 N$ & $0.68 N$
\end{tabular}

TABle 1. Asymptotic estimates of the condition numbers for different boundary equations and different geometries.

(1997). Remarkably, the condition numbers for the L-shaped domain are essentially the same as for the square. The results for the last two problems indicate that the double-layer formulation possess good spectral properties even for more challenging problems in which periodicity is locally violated. However, at this stage our understanding of the spectral properties for such problems is incomplete.

In passing, we note that although the condition numbers for the single layer potential perform somewhat poorly, the estimates in the tables can be improved by a factor of 10 using Wieland's deflation technique.

\section{REFERENCES}

[1] K. E. Atkinson. The numerical solution of integral equations of the second kind. Cambridge University Press, Cambridge, 1997.

[2] R. J. Duffin. Discrete potential theory. Duke Mathematical Journal, 20:233-251, 1953.

[3] P. G. Martinsson. Fast multiscale methods for lattice equations. Ph.D. thesis, The University of Texas at Austin, Computational and Applied Mathematics, 2002.

[4] S. G. Mikhlin. Integral equations and their applications to certain problems in mechanics, mathematical physics and technology. Pergamon Press, New York, 1957. Translated from the Russian by A. H. Armstrong.

Texas Institute for Computational and Applied Mathematics, The University of Texas at Austin, Austin TX 78712, USA 